\section{Was liegt wirklich in der Luft?}

\author{
Zu wissen, welche Allergene in der Luft enthalten sind, ist für Aller- \\ giekranke natürlich relevant. Klinische Effekte könnten aber auch \\ biogene Partikel haben, die einen nennenswerten Anteil des \\ Gesamtaerosols ausmachen.
}

W enn Patienten über gesundheitliche Beeinträchtigungen in schimmelpilzbelasteten Gebäuden klagen, für die es bisher keine wissenschaftliche Erklärung gab, könnten biogene Partikel dahinter stecken. Die kleinen Partikel sind omnipräsent und werden somit auch beim Hausbau mit in die Mauern eingearbeitet.

\section{Biomasse mit ungeklärter Relevanz}

Der hohe Anteil unbekannter Substanzen, der bei chemischen Analysen von Aerosolen meist übrig bleibt, könnte biogener Natur sein, erklärte Prof. Dr. Ruprecht Jänicke vom Institut für Physik der Atmosphäre der Universität Mainz. Zum biogenen Aerosol zählen beispielsweise zelluläre Partikel, Pflanzenteile, Pollen, Viren und Bakterien. Jänicke stellte in seinen Untersuchungen fest, dass der biologische Anteil am Gesamtaerosol in Stadtgebieten 10 bis 30\% ausmachen kann, in dicht bewachsenen Gebieten ist der Anteil vermutlich deutlich höher. Entgegen den allgemeinen Vermutungen unterliegen die zellulären Partikel aber keinen jahreszeitlichen Schwankungen.

Biogene Partikel haben durchaus Bedeutung für Umwelt und Gesundheit. Jänicke machte darauf aufmerksam, dass sie möglicherweise das Klima beeinflussen. Pollen können beispielsweise sehr viel Wasser aufnehmen, bis zum dreifachen ihrer eigenen Masse, und damit die Bildung von Wolken fördern. Andere Mikroorganismen wiederum dienten als Kristallisationskeime für die Bildung von Eis.

Welche gesundheitlichen Effekte das biogene Material auslösen kann, ist noch nicht eindeutig geklärt. Näher untersucht hat Jänicke Fragmente der Schimmelpilze Aspergillus versicolor, Penicillium melinii und Cladosporium cladosporioides. Nicht nur die Sporen gehen in Aerosole über, sondern auch in noch wesentlich höherer Zahl Fragmente dieser Schimmelpilze. Sie könnten also auch in großen Mengen eingeatmet werden. Interessant dabei ist, dass Pilzfragmente dieselben Antigenstrukturen tragen wie die entsprechenden Sporen, was auf die potenzielle allergologische Relevanz der Bioaerosole hindeutet.

\section{Gutes Klima für Pollen}

Die postulierte Erwärmung des Erdklimas könnte auch für Allergiker erhebliche Konsequenzen nach sich ziehen. Das Intergovernmental Panel on Climate Change in Genf (www.ipcc.ch) verzeichnete zwischen 1860 und 2005 einen Anstieg der durchschnittlichen globalen Temperatur um $0,49^{\circ} \mathrm{C}$. Diese Erwärmung spielte sich vorwiegend auf der nördlichen Erdhalbkugel ab.

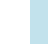

Priv.-Doz. Dr. Annette Menzel von der Technischen Universität München erklärte, dass der Klimawandel auch die Vegetation beeinflusst. So verlängere sich die Vegetationsperiode Jahr für Jahr um im Mittel 0,35 Tage. In einer groß angelegten Metanalyse stellte Menzel fest, dass die Blatt- und Blütenbildung in Europa durchschnittlich 2,5 Tage pro Dekade früher beginnt. Obst reift im Durchschnitt 2,4 Tage pro Dekade früher, und das Laub verfärbt sich im Herbst 0,2 Tage pro Dekade später.

Das bedeutet für viele Allergiker ein verlängertes Leiden. In den letzten Jahren war die Pollensaison durchschnittlich elf Tage länger als Mitte des letzten Jahrhunderts. Menzel zeigte anhand verschiedener Studiendaten, dass die Pollensaison immer früher beginnt und immer später aufhört. Der Pollenflugkalender ist also nicht stabil. Außerdem produzieren einige Pflanzen mehr Pollen als früher, zum Beispiel Gräser, und einige Pollen besitzen eine stärkere allergene Wirkung.

jn

Session „Aerobiological“. $5^{\text {th }}$ Symposium on Environmental Allergy and Allergotoxicology: Aerosols in the Initiation of Allergy, München, 8.-9. Dezember 2006

\begin{abstract}
Birke ist nicht gleich Birke Pollenflugvorhersagen sind für Allergiker ein wichtiges Instrument, um Allergene zu meiden oder vorbeugend Medikamente einzunehmen. Doch gibt es Verbesserungsbedarf. Zum einen sagen die Prognosen nichts über die individuelle Allergenexposition aus, zum anderen ist unklar, ob die Pollenmenge mit der Allergenmenge korreliert. Priv.Doz. Dr. Jeroen Buters, Zentrum für Allergie und Umwelt (ZAUM) der Technischen Universität München, wies darauf hin, dass Birkenpollen mal mehr, mal weniger Bet $v 1$ enthalten. In eigenen Untersuchungen beobachtete er, dass die Menge an Bet v 1 von Jahr zu Jahr variiert. Auch fanden sich an Sammelstellen in München und in Nordrhein-Westfalen unterschiedliche Allergenmengen. Die Konzentration an Bet v 1 kann von Pollen zu Pollen bis zum Faktor acht differieren. Ein auffallend großer Unterschied war vor allem am Saisonende zu beobachten, wenn nur noch wenige Birkenpollen vorhanden waren, deren Konzentration an Bet $\mathbf{v} 1$ aber vergleichsweise hoch war.

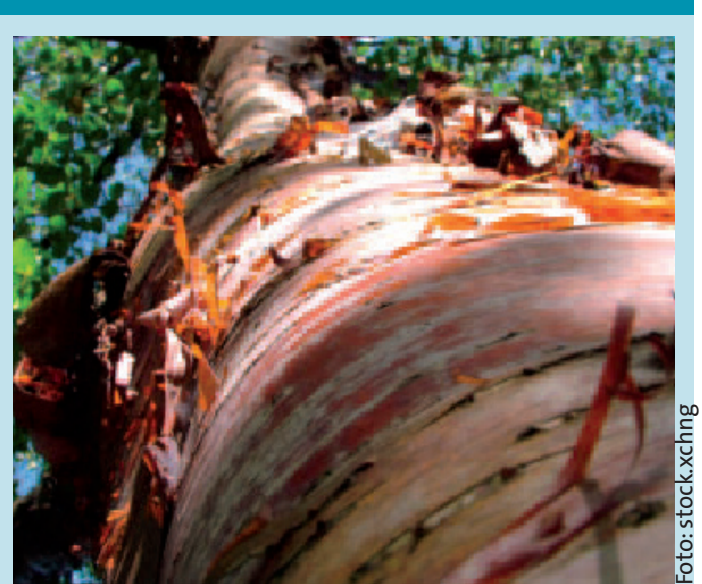

„Um eine aussagekräftige Vorhersage machen zu können, müssen wir neben den Pollenmengen auch die Allergenkonzentrationen messen“, war Buters` Schlussfolgerung. 